\title{
Group-theoretic conditions under which closed aspherical manifolds are covered by Euclidean space
}

by

Hanspeter Fischer (Muncie, IN) and David G. Wright (Provo, UT)

\begin{abstract}
Hass, Rubinstein, and Scott showed that every closed aspherical (irreducible) 3-manifold whose fundamental group contains the fundamental group of a closed aspherical surface, is covered by Euclidean space. This theorem does not generalize to higher dimensions. However, we provide geometric tools with which variations of this theorem can be proved in all dimensions.
\end{abstract}

1. Introduction and statement of results. Given a closed aspherical manifold $M$, one is interested in conditions on its fundamental group which ensure that $M$ is covered by Euclidean space.

Employing least area techniques, Hass, Rubinstein, and Scott [9] showed that this is the case when $M$ is a $P^{2}$-irreducible 3 -manifold whose fundamental group contains a subgroup isomorphic to the fundamental group of a closed surface other than $S^{2}$ or $P^{2}$. It is a long-standing conjecture that all irreducible closed aspherical 3-manifolds are covered by Euclidean space.

Davis [3] constructed examples that answered the higher-dimensional conjecture in the negative. In fact, Davis's exotic manifolds illustrate that the Hass-Rubinstein-Scott Theorem does not generalize to higher dimensions. His open contractible manifolds (of any given dimension greater than three) are not homeomorphic to Euclidean space, although each of them covers a closed manifold $M$ whose fundamental group contains a subgroup isomorphic to the fundamental group of a closed codimension-one manifold $N$ which is covered by Euclidean space.

Independently, Houghton [10] and Jackson [11] proved the following theorem (see Sections 2 and 3 for definitions):

THEOREM 1. Let

$$
1 \rightarrow H \rightarrow G \rightarrow Q \rightarrow 1
$$

2000 Mathematics Subject Classification: 57N99, 57S30, 20 F99.

The first-named author would like to thank the Department of Mathematics at Brigham Young University for its summer research support. 
be a short exact sequence of finitely presented infinite groups. If either $H$ or $Q$ is one-ended, then $G$ is simply connected at infinity.

The universal covering space of a closed aspherical manifold $M$ is homeomorphic to Euclidean space if it is simply connected at infinity (provided we assume irreducibility if the manifold is 3 -dimensional). Since this is the case precisely if its fundamental group $G=\pi_{1}(M)$ is simply connected at infinity, Theorem 1 implies that $M$ will be covered by Euclidean space if one can exhibit a finitely presented infinite normal subgroup $H$ of $G$ of infinite index such that either $H$ or $G / H$ is one-ended. (Note that the fundamental group of a compact manifold is always finitely presented.)

In this article, we will develop tools that yield a geometric proof of the Houghton-Jackson Theorem. We will also show how one can use these same tools to prove related results. Some of the theorems, for which we provide alternative proofs, are known. However, our techniques allow us to establish results that were not previously known.

One of the theorems we shall prove in this way is

Theorem 2. Let $M$ be a closed aspherical n-manifold (irreducible if $n=3)$. Suppose the fundamental group of $M$ contains a non-trivial cyclic normal subgroup. Then $M$ is covered by Euclidean space.

REMARK. We note that such a subgroup must, in fact, be infinite cyclic.

In dimensions $n \geq 5$, Theorem 2 has been proved by Lee and Raymond [12], using algebraic techniques. In dimension $n=3$, Theorem 2 can also be deduced from the Seifert fiber space conjecture, whose proof was completed only recently by Gabai [8], and, independently, by Casson and Jungries [2].

Combining Theorems 1 and 2 (see Section 6), one obtains a HassRubinstein-Scott-like result:

Corollary A. Let $N$ and $M$ be closed aspherical manifolds of dimension $k$ and $n$, respectively, with $k<n$ (and $M$ irreducible if $n=3$ ). If $\pi_{1}(N)$ is isomorphic to a normal subgroup of $\pi_{1}(M)$, then $M$ is covered by Euclidean space.

We will then relax the normality condition and establish

TheOREM 3. Let $H$ be a finitely presented subgroup of a finitely presented group $G$. Suppose that the index of $H$ in its normalizer $\mathbb{N}_{G}(H)$ in $G$ is infinite. If both $H$ and $G$ are one-ended and the pair $(G, H)$ is two-ended, then $G$ is simply connected at infinity.

Applied to the setting of aspherical manifolds, Theorem 3 implies the following alternative to Corollary A.

Corollary B. Let $N$ and $M$ be closed orientable aspherical manifolds of dimension $n-1$ and $n$, respectively (with $M$ irreducible if $n=3$ ). If 
$\pi_{1}(N)$ is isomorphic to a subgroup of $\pi_{1}(M)$ which has infinite index in its normalizer in $\pi_{1}(M)$, then $M$ is covered by Euclidean space.

The fact that the above assumptions ensure the two-endedness of the pair $\left(\pi_{1}(M), \pi_{1}(N)\right)$ follows from a theorem by Swarup [17].

In the last section of this article, we will analyze Davis's examples from the viewpoint of Corollary B. We will show that the situation is prototypical of the obstruction which one encounters, by verifying that $\pi_{1}(N)$ equals its normalizer in $\pi_{1}(M)$ in these examples.

2. Definitions. We begin by reviewing some basic definitions. Recall that if $p: \bar{X} \rightarrow X$ is a covering map (of connected, locally path connected topological spaces), then the group $\operatorname{Aut}(\bar{X} \rightarrow X)$ of covering transformations is isomorphic to $\mathbb{N}_{G}(H) / H$, where $H=p_{\#}\left(\pi_{1}(\bar{X})\right), G=\pi_{1}(X)$, and $\mathbb{N}_{G}(H)$ denotes the normalizer of $H$ in $G$. We will always suppress base points. The action of $\operatorname{Aut}(\bar{X} \rightarrow X)$ on $\bar{X}$ is properly discontinuous and fixed-point free. (Since all our spaces will be locally compact and Hausdorff, we will call the action of a group $Q$ on a topological space $Y$ properly discontinuous if $\{g \in Q \mid g(C) \cap C \neq \emptyset\}$ is finite for every compact subset $C \subseteq Y$.) If $X$ has a universal covering space, we will denote it by $\widetilde{X}$.

Conversely, if a group $G$ acts on a connected, locally path connected topological space $Y$ properly discontinuously and fixed-point free, then the quotient map $Y \rightarrow Y / G$ is a regular covering projection with automorphism group isomorphic to $G$.

We will call the action of a group $H$ on a topological space $X$ cocompact if there is a compact subset $C \subseteq X$ such that $H(C)=X$. Here, and later, $H(E)$ is defined to be $\bigcup\{h(E) \mid h \in H\}$ for subsets $E \subseteq X$.

A non-compact topological space $Y$ is called one-ended if for every compact set $A \subseteq Y$ there is a compact set $B \subseteq Y$ such that $A \subseteq B$ and every pair of points in $Y \backslash B$ is joined by a path in $Y \backslash A$. A one-ended space $Y$ is called simply connected at infinity if for every compact set $A \subseteq Y$ there is a compact set $B \subseteq Y$ such that $A \subseteq B$ and loops in $Y \backslash B$ contract in $Y \backslash A$. In the next section, we will extend these definitions to groups. Two-endedness of pairs of groups will be defined in Section 7 .

A topological space $Y$ is called locally simply connected if for every $y \in Y$ and every neighborhood $U$ of $y$ in $Y$ there is a neighborhood $V$ of $y$ in $Y$ such that $V \subseteq U$ and loops in $V$ contract in $U$.

A manifold is called aspherical if its universal covering space is contractible. We note that all open contractible manifolds of dimension at least two are one-ended. Moreover, if an open contractible $n$-manifold is simply connected at infinity, then it is homeomorphic to Euclidean space, provided we assume that the manifold is irreducible in case $n=3$. (For $n=3$, see 
Wall [19] and Brown [1]; for $n=4$, this is due to Freedman [7]; for $n \geq 5$ we have the result of Stallings [16] and its strengthening by Siebenmann [15].) Clearly, all one-dimensional and two-dimensional closed aspherical manifolds are covered by Euclidean space.

We add to this list of definitions some relative notions of connectivity. Let a triple $C \subseteq D \subseteq Y$ of topological spaces be given. We will from now on say that $C$ is path connected in $D$ if every pair of points in $C$ is joined by a path in $D$. Similarly, if all loops in $C$ contract in $D$, we will call $C$ simply connected in $D$. We will say that $C$ is one-ended in $D$ with respect to $Y$ if $C$ is not contained in a compact subset of $Y$ and for every compact set $A \subseteq Y$ there is a compact set $B \subseteq Y$ such that $A \subseteq B$ and every pair of points in $C \backslash B$ is joined by a path in $D \backslash A$. Similarly, $C$ is simply connected at infinity in $D$ with respect to $Y$ if $C$ is not contained in a compact subset of $Y$ and for every compact set $A \subseteq Y$ there is a compact set $B \subseteq Y$ such that $A \subseteq B$ and loops in $C \backslash B$ contract in $D \backslash A$. Whenever the ambient space $Y$ is understood, we drop the reference to it.

3. Some tools. For this section, we fix two topological spaces $X$ and $Y$ which are connected, locally path connected (and hence path connected), locally compact, and Hausdorff. Suppose $H$ is a subgroup of a group $G$ and assume that $H$ and $G$ act properly discontinuously on the spaces $X$ and $Y$, respectively. Suppose, further, that the action of $H$ on $X$ is cocompact. We will also assume that $Y$ is locally simply connected (although this is irrelevant for Lemmas 1, 3, and 4).

REMARK. The existence of an action of $H$ on a space $X$ which satisfies the above hypotheses is equivalent to the fact that $H$ is finitely generated.

We state the following lemma for the record, its proof is immediate.

Lemma 1. For every compact set $C \subseteq Y$ there is a compact set $D \subseteq Y$ such that $C \subseteq D$ and $C$ is path connected in $D$.

Lemma 2. Suppose $Y$ is simply connected. Then for every compact set $C \subseteq Y$ there is a compact set $D \subseteq Y$ such that $C \subseteq D$ and $C$ is simply connected in $D$.

Proof. Choose open subsets $U_{0}, U_{1}, \ldots, U_{k}, V_{0}, V_{1}, \ldots, V_{k}$ of $Y$ such that $C \subseteq \bigcup\left\{U_{i} \mid i=0,1, \ldots, k\right\}$ and, for each $i, U_{i}$ is simply connected in $V_{i}$, and $V_{i}$ has compact closure. One can then find a finite collection $\mathcal{W}$ of open path connected subsets of $Y$ such that $C \subseteq \cup \mathcal{W}$ and with the property that for each pair $W_{1}, W_{2} \in \mathcal{W}$ with $W_{1} \cap W_{2} \neq \emptyset$, there is a $U_{i}$ with $W_{1} \cup W_{2} \subseteq U_{i}$.

For example, one could use the following partition of unity to find $\mathcal{W}$ : choose a compact set $E \subseteq Y$ with $C \subseteq \operatorname{int} E \subseteq E \subseteq \bigcup\left\{U_{i} \mid i=0,1, \ldots, k\right\}$ and continuous functions $\phi_{i}: E \rightarrow[0,1]$ such that $\phi_{i}^{-1}((0,1]) \subseteq U_{i}$ for all 
$i=0,1, \ldots, k$ and $\sum_{i=0}^{k} \phi(x)=1$ for each $x \in E$. Define a map $f$ from $E$ to a $k$-simplex $\sigma_{k}=\left\langle v_{0}, v_{1}, \ldots, v_{k}\right\rangle$ by defining $f(x)=\sum_{i=0}^{k} \phi_{i}(x) v_{i}$ for $x \in E$. Let $\mathcal{W}^{\prime}$ be a covering of $\sigma_{k}$ by finitely many open sets so that for each pair $W_{1}^{\prime}, W_{2}^{\prime} \in \mathcal{W}^{\prime}$ with $W_{1}^{\prime} \cap W_{2}^{\prime} \neq \emptyset, W_{1}^{\prime} \cup W_{2}^{\prime}$ lies in the open star $S_{i}$ of $v_{i}$ in $\sigma_{k}$ for some $i$. Since $f^{-1}\left(S_{i}\right) \subseteq U_{i}$, one can now select the desired collection $\mathcal{W}$ from the path components of the sets $f^{-1}\left(W^{\prime}\right) \cap \operatorname{int} E, W^{\prime} \in \mathcal{W}^{\prime}$.

Define a finite graph $\Gamma$ as follows. For each $W \in \mathcal{W}$ take a vertex $v(W)$. Join two distinct vertices $v(W)$ and $v\left(W^{\prime}\right)$ by an edge $e\left(W, W^{\prime}\right)$ whenever $W \cap W^{\prime} \neq \emptyset$. Choose a map $\mu: \Gamma \rightarrow Y$ such that $\mu(v(W)) \in W$ and $\mu\left(e\left(W, W^{\prime}\right)\right) \subseteq W \cup W^{\prime}$ for all $W, W^{\prime} \in \mathcal{W}$. Since $Y$ is simply connected, there is a homotopy from $\mu$ to a constant map. Choose a compact set $D$ such that it contains the closure of each $V_{i}$ and the image of this homotopy. A loop $\alpha$ in $C$ can now be subdivided into paths $\alpha_{i}$ so that each $\alpha_{i}$ lies in an element $W_{i} \in \mathcal{W}$. If we connect the endpoints of each $\alpha_{i}$ to $\mu\left(v\left(W_{i}\right)\right)$ with a path in $W_{i}$, we produce a bootstrap pattern between $\alpha$ and $\Gamma$ whose loops lie alternately in a member of $\mathcal{W}$ and in the union of two intersecting members of $\mathcal{W}$. This allows us to homotope $\alpha$ into $\mu(\Gamma)$ within $D$. From there we can contract it to a point within $D$.

Lemma 3. For every compact set $C \subseteq Y$ there is a compact set $D \subseteq Y$ such that $C \subseteq D$ and $H(C)$ is path connected in $H(D)$.

The proof is similar to but simpler than the proof of

Lemma 4. If $X$ is one-ended, then for every compact set $C \subseteq Y$ there is a compact set $D \subseteq Y$ such that $C \subseteq D$ and $H(C)$ is one-ended in $H(D)$.

Proof. Let a compact set $C \subseteq Y$ be given. Choose a compact set $E \subseteq X$ so that $H($ int $E)=X$. Choose a compact set $D^{\prime} \subseteq Y$ such that $C \subseteq D^{\prime}$ and $g_{1}\left(D^{\prime}\right) \cap g_{2}\left(D^{\prime}\right) \neq \emptyset$ whenever $g_{1}, g_{2} \in H$ and $g_{1}(E) \cap g_{2}(E) \neq \emptyset$. (This is possible since the set $\{g \in H \mid E \cap g(E) \neq \emptyset\}$ is finite.) Choose a compact set $D \subseteq Y$ such that $D^{\prime} \subseteq D$ and $D^{\prime}$ is path connected in $D$.

Now, let $A \subseteq Y$ be compact. Choose a compact set $L \subseteq X$ such that $X \backslash L$ is path connected in $X \backslash \bigcup\{g(E) \mid g \in H, g(D) \cap A \neq \emptyset\}$. Define the compact set $B=\bigcup\{g(D) \mid g \in H$ and either $g(E) \cap L \neq \emptyset$ or $g(D) \cap A \neq \emptyset\}$.

If $a, b \in H(C) \backslash B$, then there are $g_{a}, g_{b} \in H$ such that $a \in g_{a}\left(D^{\prime}\right)$ and $b \in g_{b}\left(D^{\prime}\right)$. Hence, $g_{a}(D) \cap A=\emptyset, g_{a}(E) \cap L=\emptyset, g_{b}(D) \cap A=\emptyset$, and $g_{b}(E) \cap L=\emptyset$. Pick a point $a^{\prime} \in g_{a}(E)$, a point $b^{\prime} \in g_{b}(E)$, and choose a path $\gamma^{\prime}:[0,1] \rightarrow X \backslash \bigcup\{g(E) \mid g \in H, g(D) \cap A \neq \emptyset\}$ with $\gamma^{\prime}(0)=a^{\prime}$ and $\gamma^{\prime}(1)=b^{\prime}$. Choose $n \in \mathbb{N}$ such that for each $i \in\{0,1, \ldots, n-1\}$ there is a $g_{i} \in H$ such that $\gamma^{\prime}\left(\left[\frac{i}{n}, \frac{i+1}{n}\right]\right) \subseteq g_{i}($ int $E)$. Then we have $g_{a}\left(D^{\prime}\right) \cap g_{0}\left(D^{\prime}\right) \neq \emptyset$, $g_{b}\left(D^{\prime}\right) \cap g_{n-1}\left(D^{\prime}\right) \neq \emptyset$, and $g_{i}\left(D^{\prime}\right) \cap g_{i+1}\left(D^{\prime}\right) \neq \emptyset$ for all $i \in\{0,1, \ldots, n-2\}$, but $g_{i}(D) \cap A=\emptyset$ for all $i \in\{0,1, \ldots, n-1\}$. Since $D^{\prime}$ is path connected in $D$, there is a path $\gamma:[0,1] \rightarrow H(D) \backslash A$ with $\gamma(0)=a$ and $\gamma(1)=b$. 
Lemma 5. Suppose both $X$ and $Y$ are simply connected. Then for every compact set $C \subseteq Y$ there is a compact set $D \subseteq Y$ such that $C \subseteq D$ and $H(C)$ is simply connected in $H(D)$.

REMARK. The existence of an action of $H$ on a simply connected space $X$ which satisfies the additional hypotheses stated at the beginning of this section is equivalent to the fact that $H$ is finitely presented.

Proof. Let $C \subseteq Y$ be compact. Choose a compact set $C^{\prime} \subseteq Y$ such that $C \subseteq \operatorname{int} C^{\prime}$. Choose a compact set $E \subseteq X$ such that $H(\operatorname{int} E)=X$ and $g_{1}(E) \cap g_{2}(E) \neq \emptyset$ whenever $g_{1}, g_{2} \in H$ and $g_{1}\left(C^{\prime}\right) \cap g_{2}\left(C^{\prime}\right) \neq \emptyset$. Choose a compact set $E^{\prime} \subseteq X$ such that $E \subseteq E^{\prime}$ and $E$ is path connected in $E^{\prime}$. Choose a compact set $F \subseteq Y$ such that $C^{\prime} \subseteq F$ and $\bigcap\{g(F) \mid g \in S\} \neq \emptyset$ whenever $S \subseteq H$ and $\bigcap\left\{g\left(E^{\prime}\right) \mid g \in S\right\} \neq \emptyset$. Choose a compact set $F^{\prime} \subseteq Y$ such that $F \subseteq F^{\prime}$ and $F$ is path connected in $F^{\prime}$. Put $F^{\prime \prime}=\bigcup\left\{g\left(F^{\prime}\right) \mid g \in H\right.$, $F^{\prime} \cap h\left(F^{\prime}\right) \neq \emptyset$, and $h\left(F^{\prime}\right) \cap g\left(F^{\prime}\right) \neq \emptyset$ for some $\left.h \in H\right\}$.

By Lemma 2, there is a compact subset $D \subseteq Y$ such that $F^{\prime \prime} \subseteq D$ and $F^{\prime \prime}$ is simply connected in $D$. For $n \in \mathbb{N}$ and $x \in[0,1]^{2}$ define the sets

$$
\begin{aligned}
\mathcal{G}(n)= & \left\{\left\{\frac{i}{n}\right\} \times\left[\frac{j}{n}, \frac{j+1}{n}\right] \mid i \in\{0,1, \ldots, n\}, j \in\{0,1, \ldots, n-1\}\right\} \\
& \cup\left\{\left[\frac{i}{n}, \frac{i+1}{n}\right] \times\left\{\frac{j}{n}\right\} \mid i \in\{0,1, \ldots, n-1\}, j \in\{0,1, \ldots, n\}\right\}, \\
\mathcal{B}(n)= & \left\{P \in \mathcal{G}(n) \mid P \subseteq \partial[0,1]^{2}\right\}, \quad \mathcal{I}(n)=\mathcal{G}(n) \backslash \mathcal{B}(n), \\
\mathcal{N}(x, n)= & \{P \in \mathcal{G}(n) \mid x \in P\}, \\
\mathcal{D}(n)= & \left\{\frac{1}{n}, \frac{2}{n}, \ldots, \frac{n-1}{n}\right\} \times\left\{\frac{1}{n}, \frac{2}{n}, \ldots, \frac{n-1}{n}\right\} .
\end{aligned}
$$

Let $\gamma: \partial[0,1]^{2} \rightarrow H(C)$ be a loop. Since $\gamma\left(\partial[0,1]^{2}\right) \subseteq H\left(\right.$ int $\left.C^{\prime}\right)$, there is an $n \in \mathbb{N}$ such that for all $P \in \mathcal{B}(n)$ there is a $g_{P} \in H$ with $\gamma(P) \subseteq$ $g_{P}\left(\right.$ int $\left.C^{\prime}\right)$. Choose $\gamma^{\prime}: \partial[0,1]^{2} \rightarrow X$ such that $\gamma^{\prime}(P) \subseteq g_{P}\left(E^{\prime}\right)$ for all $P \in$ $\mathcal{B}(n)$. Since $X$ is simply connected, we can extend $\gamma^{\prime}$ to $f^{\prime}:[0,1]^{2} \rightarrow X$. Since $H\left(\right.$ int $\left.E^{\prime}\right)=X$, there is an $m \in \mathbb{N}(m \geq 2)$ such that for all $P \in \mathcal{G}(n m)$ there is a $g_{P} \in H$ with $f^{\prime}(P) \subseteq g_{P}\left(\right.$ int $\left.E^{\prime}\right)$.

Extend $\gamma$ to a map $f: \partial[0,1]^{2} \cup \mathcal{D}(n m) \rightarrow H(F)$ such that $f(x) \in$ $\bigcap\left\{g_{P}(F) \mid P \in \mathcal{N}(x, n m)\right\}$ for all $x \in \mathcal{D}(n m)$. Next, extend $f$ to a map $\bigcup \mathcal{G}(n m) \rightarrow H\left(F^{\prime}\right)$ such that for all $P \in \mathcal{I}(n m)$ with $P \subseteq \operatorname{int}[0,1]^{2}$ we have $f(P) \subseteq g_{P}\left(F^{\prime}\right)$, and for all $P \in \mathcal{I}(n m)$ with $P \nsubseteq \operatorname{int}[0,1]^{2}$ we have $f(P) \subseteq g_{P}\left(F^{\prime}\right) \cup g_{Q}\left(F^{\prime}\right)$ for some $Q \in \mathcal{B}(n)$ with $P \cap Q \neq \emptyset$. Finally, since for all $i, j \in\{0,1, \ldots, n m-1\}$ there is a $g \in H$ such that $f\left(\partial\left(\left[\frac{i}{n m}, \frac{i+1}{n m}\right] \times\right.\right.$ $\left.\left.\left[\frac{j}{n m}, \frac{j+1}{n m}\right]\right)\right) \subseteq g\left(F^{\prime \prime}\right)$, we can, by choice of $D$, extend $f$ to a map $[0,1]^{2} \rightarrow$ $H(D)$. Hence, $\gamma$ contracts in $H(D)$.

Similarly, we have 
Lemma 6. Suppose $X$ is simply connected at infinity and $Y$ is simply connected. Then for every compact set $C \subseteq Y$ there is a compact set $D \subseteq Y$ such that $C \subseteq D$ and $H(C)$ is simply connected at infinity in $H(D)$.

Proof. We have to change the proof of Lemma 5 only slightly. Using the same setup, let $A \subseteq Y$ be compact. Choose a compact set $L \subseteq X$ so that $X \backslash L$ is simply connected in $X \backslash \bigcup\left\{g\left(E^{\prime}\right) \mid g \in H, g(D) \cap A \neq \emptyset\right\}$. Set $B=A \cup \bigcup\left\{g\left(C^{\prime}\right) \mid g \in H, g\left(E^{\prime}\right) \cap L \neq \emptyset\right\}$. Now, if $\gamma: \partial[0,1]^{2} \rightarrow H(C) \backslash B$ is a loop, then $\gamma^{\prime}\left(\partial[0,1]^{2}\right) \subseteq X \backslash L$ (where $\gamma^{\prime}$ is as before) and $\gamma^{\prime}$ extends to a map $f^{\prime}:[0,1]^{2} \rightarrow X \backslash \bigcup\left\{g\left(E^{\prime}\right) \mid g \in H, g(D) \cap A \neq \emptyset\right\}$. This yields an extension $f:[0,1]^{2} \rightarrow H(D) \backslash A$ of $\gamma$.

Let us recall the following standard terminology. The group $H$ is called one-ended if $X$ is one-ended, and simply connected at infinity if $X$ is locally simply connected, simply connected and simply connected at infinity. It is well known that these definitions do not depend on the choice of $X$, but rather are invariants of the group $H$. Note that admissible choices for $X$ include (appropriate) locally finite $\mathrm{CW}$-complexes and topological manifolds.

REMARK. Lemmas 4 and 6 also provide an alternative to the standard proofs of the fact that the above definitions are independent of the choice of $X$. For if we further assume the action of $H$ on $Y$ to be cocompact, the conclusions of Lemmas 4 and 6 are equivalent to $Y$ being one-ended and simply connected at infinity, respectively.

Lemma 7. Suppose both $X$ and $Y$ are simply connected, that the action of $G$ on $Y$ is also cocompact, and that $H$ is a normal subgroup of $G$. Then for every compact set $A \subseteq Y$ there is a compact set $B \subseteq Y$ with $A \subseteq B$ such that if $\gamma$ is a loop in $Y \backslash H(B)$ and $h \in H$, we can homotope $\gamma$ to $h \circ \gamma$ by a homotopy missing $H(A)$.

Proof. Let the compact set $A \subseteq Y$ be given. Choose a compact set $C \subseteq Y$ such that $G(\operatorname{int} C)=Y$. By Lemma 3 , there is a compact set $D \subseteq Y$ such that $C \subseteq D$ and $H(C)$ is path connected in $H(D)$. Define $E=\bigcup\{g(D) \mid$ $g \in G, D \cap g(D) \neq \emptyset\}$. Then $E$ is a compact subset of $Y$ containing $D$. Using Lemma 5, we may choose a compact set $F \subseteq Y$ such that $E \subseteq F$ and $H(E)$ is simply connected in $H(F)$. Put $B=\bigcup\{g(F) \mid g \in G, A \cap g(F) \neq \emptyset\}$.

Now, let $\gamma:[0,1] \rightarrow Y \backslash H(B)$ be a map with $\gamma(0)=\gamma(1)$ and let $h \in H$. Choose $n \in \mathbb{N}$ such that for all $i \in\{0,1, \ldots, n-1\}$ there is a $g_{i} \in G$ with $\gamma\left(\left[\frac{i}{n}, \frac{i+1}{n}\right]\right) \subseteq g_{i}(\operatorname{int} C)$. Then both $\gamma\left(\left[\frac{i}{n}, \frac{i+1}{n}\right]\right)$ and $h \circ \gamma\left(\left[\frac{i}{n}, \frac{i+1}{n}\right]\right)$ are contained in $g_{i} H(C)$ for all $i \in\{0,1, \ldots, n-1\}$. For each $i \in\{0,1, \ldots, n-1\}$ we connect $\gamma\left(\frac{i}{n}\right)$ to $h \circ \gamma\left(\frac{i}{n}\right)$ with a path in $g_{i} H(D)$. For $i \in\{0,1, \ldots, n-1\}$ and $j=i+1$, we have $g_{i}(D) \cap g_{j}(D) \neq \emptyset$, where we put $g_{n}=g_{0}$. Therefore, for all $h^{\prime} \in H$ there is an $h^{\prime \prime} \in H$ such that $g_{i} h^{\prime \prime}(D) \cap g_{j} h^{\prime}(D) \neq \emptyset$, because $H$ is normal in $G$, so that $g_{j} h^{\prime}(D) \subseteq g_{i} h^{\prime \prime}(E)$ by definition of $E$. We conclude that 
$g_{j} H(D) \subseteq g_{i} H(E)$. Consequently, the two paths joining $\gamma\left(\frac{i}{n}\right)$ to $h \circ \gamma\left(\frac{i}{n}\right)$ and $\gamma\left(\frac{j}{n}\right)$ to $h \circ \gamma\left(\frac{j}{n}\right)$, respectively, both lie in $g_{i} H(E)$, as do the paths $\left.\gamma\right|_{\left[\frac{i}{n}, \frac{j}{n}\right]}$ and $\left.h \circ \gamma\right|_{\left[\frac{i}{n}, \frac{j}{n}\right]}$. By choice of $F$, these loops can be contracted in $g_{i} H(F)$.

This fills in a homotopy from $\gamma$ to $h \circ \gamma$ missing $H(A)$. For if $g_{i} H(F) \cap$ $H(A) \neq \emptyset$ for some $i \in\{0,1, \ldots, n-1\}$, we would have $g_{i} h^{\prime}(F) \cap A \neq \emptyset$ for some $h^{\prime} \in H$, so that $g_{i} h^{\prime}(F) \subseteq B$, by the definition of $B$, implying that $\gamma\left(\left[\frac{i}{n}, \frac{i+1}{n}\right]\right) \subseteq g_{i}(C) \subseteq g_{i}(F) \subseteq \bar{H}(B)$, contrary to our assumption on $\gamma$.

Later, we will also need

LEMmA 8. Let $L$ be a locally finite simplicial complex, $r: \widetilde{L} \rightarrow L$ the universal covering, $U$ a connected subcomplex of $L$ such that the inclusion induced homomorphism $\pi_{1}(U) \rightarrow \pi_{1}(L)$ is surjective, and $P$ a finite subcomplex of $L$. Then for every compact set $A \subseteq \widetilde{L}$ there is a compact set $B \subseteq \widetilde{L}$ with $A \subseteq B$ and such that loops in $r^{-1}(U \cup P) \backslash B$ can be homotoped into $r^{-1}(U)$ with a homotopy in $\widetilde{L}$ missing $A$.

Proof. Since $\pi_{1}(U) \rightarrow \pi_{1}(L)$ is surjective and $U$ is connected, there is a homotopy $H$ that takes the 1-skeleton of $L$ into $U$ leaving the 1-simplices of $U$ fixed. For each 1-simplex $\sigma$ of the finitely many 1-simplices of $P$ which do not lie in $U$, choose a compact subset $E_{\sigma}$ of $\widetilde{L}$ which contains a lift of the given homotopy that takes $\sigma$ into $U$. Put $E=\bigcup E_{\sigma}$. Let a compact set $A \subseteq \widetilde{L}$ be given. Choose a compact set $B^{\prime} \subseteq \widetilde{L}$ with $A \subseteq B^{\prime}$ such that every translate of $E$ (under a covering translation) which intersects $A$, lies in $B^{\prime}$. Choose a compact set $B \subseteq \widetilde{L}$ with $B^{\prime} \subseteq B$ such that every simplex of $\widetilde{L}$ which meets $B^{\prime}$, lies in $B$. Let $\alpha$ be a loop in $r^{-1}(U \cup P) \backslash B$. We can homotope $r \circ \alpha$ to a loop that lies in the union of $U$ and the 1-skeleton of $P$ such that during the homotopy points do not leave the top-dimensional simplex containing them. The lift of this homotopy lies in $\widetilde{L} \backslash B^{\prime}$; call its end $\alpha^{\prime}$. Now, $r \circ \alpha^{\prime}$ can be homotoped into $U$ using the homotopy $H$. We lift this homotopy to a homotopy of $\alpha^{\prime}$ and call its end $\alpha^{\prime \prime}$. If a point is moved during this final homotopy, then it must lie in a translate of $E$. Hence, the track of such a point must miss $A$.

\section{Proof of Theorem 2. From [20] we quote}

Lemma 9 (The Orbit Lemma I). Suppose $W$ is an open contractible $n$ manifold, $n \geq 3$. Let $h$ be a non-trivial homeomorphism of $W$ onto itself so that the group $H$ of homeomorphisms generated by $h$ acts without fixed points and properly discontinuously on $W$. If $C$ is a compact subset of $W$, then loops of $W$ can be homotoped off $H(C)$. Furthermore, given a compact set $A$ there is a compact set $B$ which contains $A$ so that loops in $W \backslash B$ can be homotoped off $H(C)$ by a homotopy that lies in $W \backslash A$. 
Proof of Theorem 2. Let $H=\langle h\rangle$ be a non-trivial cyclic normal subgroup of $\pi_{1}(M)$. Note that $h$ must have infinite order. (Otherwise $s=|\langle h\rangle|<\infty$ and $\widetilde{M} /\langle h\rangle$ is a finite-dimensional $K\left(\mathbb{Z}_{s}, 1\right)$, which contradicts the fact that $\mathbb{Z}_{s}$ has infinite cohomological dimension.) We assume that $n \geq 3$ and wish to show that $\widetilde{M}$ is simply connected at infinity.

Let $C \subseteq \widetilde{M}$ be compact. Use Lemma 7 to choose a compact set $E \subseteq \widetilde{M}$ such that $C \subseteq E$ and loops in $\widetilde{M} \backslash H(E)$ can be homotoped to any $H$ translate via a homotopy missing $H(C)$. By Lemma 9 , there is a compact set $D \subseteq \widetilde{M}$ such that $E \subseteq D$ and loops in $\widetilde{M} \backslash D$ can be homotoped into $\widetilde{M} \backslash H(E)$ by a homotopy missing $E$ (and hence $C$ ). Now, let $\gamma$ be a loop in $\widetilde{M} \backslash D$. By choice of $D$ we may already assume that $\gamma$ lies in $\widetilde{M} \backslash H(E)$. Since $\widetilde{M}$ is simply connected, $\gamma$ can be contracted to a point in $\widetilde{M}$. Let $\eta$ be the image of this contraction. Choose $n \in \mathbb{N}$ such that $h^{n}(\eta) \cap C=\emptyset$. We then homotope $\gamma$ to $h^{n} \circ \gamma$ with a homotopy missing $H(C)$, where it contracts missing $C$.

5. A geometric proof of Theorem 1. We now want to use our tools of Section 3 to give a geometric proof of the Houghton-Jackson Theorem.

To this end, let $K$ be a finite connected simplicial complex with fundamental group $G$. We identify $H$ with a subgroup of $G$ and $Q$ with $G / H$. Let $p: \widetilde{K} \rightarrow K$ be the universal covering and identify $G \equiv \operatorname{Aut}(\widetilde{K} \rightarrow K)$. Put $\bar{K}=\widetilde{K} / H$ with quotient (and covering) map $q: \widetilde{K} \rightarrow \bar{K}$, so that $H \equiv \operatorname{Aut}(\widetilde{K} \rightarrow \bar{K})$. We also identify $Q=G / H$ with $\operatorname{Aut}(\bar{K} \rightarrow K)$. Note that $H$ is isomorphic to the fundamental group of a finite simplicial complex, because it is finitely presented. Since $H$ acts properly discontinuously and cocompactly on the universal cover of this complex, we are in the setting of Section 3 with two simply connected spaces.

Choose a finite connected subcomplex $C$ of $\widetilde{K}$ such that $G(\operatorname{int} C)=\widetilde{K}$, $H(C)$ is path connected, and the inclusion induced homomorphism $\pi_{1}(q(C))$ $\rightarrow \pi_{1}(\bar{K}) \simeq H$ is surjective.

We inductively define the following subsets of $G / H$. Put $B_{0}=\{H\}$ and $B_{n}=\left\{g H \in G / H \mid g H(C) \cap g^{\prime} H(C) \neq \emptyset\right.$ for some $\left.g^{\prime} H \in B_{n-1}\right\}$. Then each $B_{n}$ is finite and $G / H=\bigcup B_{n}$. Finally, put $T_{n}=\bigcup\{g H(C) \mid g H \in$ $\left.B_{n} \backslash B_{n-1}\right\}$.

\section{Lemma 10. $G$ is one-ended.}

Proof. Let $D \subseteq \widetilde{K}$ be a compact set. Choose $n \in \mathbb{N}$ such that $D \subseteq$ $\bigcup_{j<n} T_{j}$ and $D \cap T_{n}=\emptyset$.

We claim that we may choose a compact set $E \subseteq \widetilde{K}$ with $D \subseteq E$ and such that for any $g_{1} H, g_{2} H \in B_{n}$, every point of $g_{1} H(C) \backslash E$ can be joined to some point of $g_{2} H(C)$ by a path missing $D$. To see how, for each of the finitely 
many pairs $g_{1} H, g_{2} H \in B_{n}$, connect $C$ to $g_{1}^{-1} g_{2}(C)$ by some path $\alpha$ in $\tilde{K}$. Then for every $h \in H, g_{1} h \circ \alpha$ connects $g_{1} h(C)$ to $g_{1} h g_{1}^{-1} g_{2}(C) \subseteq g_{2} H(C)$. Hence, for any $g_{1} H, g_{2} H \in B_{n}$, every point of $g_{1} H(C)$ can be connected to some point of $g_{2} H(C)$ by a concatenation of a translate of a path $\beta$ in $C$ and a translate of some finite collection of paths $\left(\alpha_{i}\right)$. Since only finitely many translates of $C$ and only finitely many translates of each $\alpha_{i}$ intersect $D$, we can choose $E$ as claimed.

Now, let $a, b \in \widetilde{K} \backslash E$. These points may or may not lie in $\bigcup_{j \leq n} T_{j}$. Without loss of generality, say $a \notin \bigcup_{j \leq n} T_{j}$ and $b \in g_{1} H(C)$ for some $g_{1} H$ $\in B_{n}$. Connect $a$ to a point $p_{1} \in g_{2} H(C)$ for some $g_{2} H \in B_{n} \backslash B_{n-1}$ with a path in $\widetilde{K} \backslash \bigcup_{j<n} T_{j}$. Connect $b$ to a point $p_{2} \in g_{2} H(C)$ by a path missing $D$. Finally, connect $p_{1}$ and $p_{2}$ in $g_{2} H(C)$. This yields a path from $a$ to $b$ missing $D$.

Lemma 11. Suppose $Q$ is one-ended and a compact set $E \subseteq \widetilde{K}$ is given. Then for every compact set $A \subseteq \widetilde{K}$ there is a compact set $B \subseteq \widetilde{K}$ such that $A \subseteq B$ and loops in $\widetilde{K} \backslash B$ can be homotoped off $H(E)$ by a homotopy that lies in $\widetilde{K} \backslash A$.

Proof. Since $\bar{K}$ is one-ended, there is a finite subcomplex $P$ of $\bar{K}$ and a connected subcomplex $U$ of $\bar{K}$ such that $U \cap q(E)=\emptyset$ and $\bar{K}=U \cup P$. Now, $Q$ is infinite so that $v(q(C)) \cap P=\emptyset$ for some $v \in Q$. Hence, $v(q(C)) \subseteq U$. Since $\pi_{1}(q(C)) \rightarrow \pi_{1}(\bar{K})$ is surjective, so is $\pi_{1}(v(q(C))) \rightarrow \pi_{1}(\bar{K})$. Hence $\pi_{1}(U) \rightarrow$ $\pi_{1}(\bar{K})$ is surjective. The result now follows at once from Lemma 8.

LEMMA 12. If $Q$ is one-ended, then $G$ is simply connected at infinity.

Proof. Since $H$ is infinite, we can repeat the argument used in the last paragraph of the proof of Theorem 2, substituting Lemma 11 for Lemma 9 and an appropriate element of $H$ for $h^{n}$.

LEMmA 13. If $a, b \in T_{n}$ lie in the same component of $\widetilde{K} \backslash \bigcup_{j<n} T_{j}$, then $a$ and $b$ can be joined by a path in $T_{n}$.

Proof. Join $a$ to $b$ via two paths, one lying in $\widetilde{K} \backslash \bigcup_{j<n} T_{j}$ and the other in $\bigcup_{j \leq n} T_{j}$. Since $\widetilde{K}$ is simply connected this loop contracts. The result now follows from the fact that $T_{n}$ separates $\bigcup_{j>n} T_{j}$ from $\bigcup_{j<n} T_{j}$.

If $H$ is one-ended we can choose a finite subcomplex $D$ of $\widetilde{K}$ such that $C \subseteq D$ and $H(C)$ is one-ended in $H(D)$, by Lemma 4. Let us also arrange for $H(D)$ to be path connected. We then put $T_{n}^{\prime}=\bigcup\left\{g H(D) \mid g H \in B_{n} \backslash B_{n-1}\right\}$.

LEMma 14. Suppose $H$ is one-ended. Then for every compact set $A \subseteq \widetilde{K}$ there is a compact subset $B \subseteq \widetilde{K}$ with $A \subseteq B$ such that if $a, b \in T_{n} \backslash B$ lie in the same component of $T_{n}$, then $a$ and $b$ can be joined by a path in $T_{n}^{\prime} \backslash A$. 
Proof. By Lemma 4, there is a compact set $B^{\prime} \subseteq \widetilde{K}$ with $A \subseteq B^{\prime}$ such that for all the finitely many $g H \in B_{n} \backslash B_{n-1}, g H(C) \backslash B^{\prime}$ is path connected in $g H(D) \backslash A$. Choose a compact set $B \subseteq \widetilde{K}$ with $B^{\prime} \subseteq B$ such that for any $g_{1} H, g_{2} H \in B_{n} \backslash B_{n-1}$ with $g_{1} H(C)$ and $g_{2} H(C)$ in the same component of $T_{n}$, every point of $g_{1} H(C) \backslash B$ can be joined to some point of $g_{2} H(C)$ by a path in $T_{n} \backslash B^{\prime}$.

Now suppose that $a, b \in T_{n} \backslash B$ lie in the same component of $T_{n}$. Say $b \in g H(C)$ with $g H \in B_{n} \backslash B_{n-1}$. Join $a$ to a point $p_{0} \in g H(C)$ by a path in $T_{n} \backslash B^{\prime}$. Then join $p_{0}$ to $b$ by a path in $g H(D) \backslash A$. This yields a path from $a$ to $b$ in $T_{n}^{\prime} \backslash A$.

Lemma 15. If $H$ is one-ended, then $G$ is simply connected at infinity.

Proof. Let a compact set $A \subseteq \widetilde{K}$ be given. By Lemma 7 , there is a compact set $E \subseteq \widetilde{K}$ with $A \subseteq E$ so that loops in $\widetilde{K} \backslash H(E)$ can be homotoped to any $H$-translate via a homotopy missing $H(A)$. Choose $n \in \mathbb{N}$ such that $E \subseteq \bigcup_{j<n} T_{j}^{\prime}$ and $E \cap T_{n}^{\prime}=\emptyset$. Pick any $s H \in B_{n} \backslash B_{n-1}$. Since $\pi_{1}((s H)(q(C))) \rightarrow \pi_{1}(\bar{K})$ is surjective, we can use Lemma 8 to find a compact set $F \subseteq \widetilde{K}$ with $E \subseteq F$ such that loops in $\bigcup_{j \leq n} T_{j}^{\prime} \backslash F$ can be homotoped into $s H(D)$ by a homotopy missing $E$ (and hence missing $A$ ). By Lemma 14, there is a compact set $B \subseteq \widetilde{K}$ with $F \subseteq B$ such that points in $T_{n} \backslash B$ that are in the same component of $T_{n}$ can be joined by paths in $T_{n}^{\prime} \backslash F$.

Now, let $\gamma$ be a loop in $\widetilde{K} \backslash B$. We wish to show that $\gamma$ contracts in $\widetilde{K} \backslash A$. We may assume that $\gamma$ is an edge path in the 1 -skeleton of $\widetilde{K}$. First we argue that we may assume, without loss of generality, that $\gamma$ lies either in $\bigcup_{j \leq n} T_{j}^{\prime} \backslash F$ or in $\widetilde{K} \backslash H(E)$. For if $\gamma$ intersects $\bigcup_{j<n} T_{j}$ and intersects $\bigcup_{j>n} \bar{T}_{j}$ we can cut it into finitely many subpaths that lie either in $\bigcup_{j \leq n} T_{j} \backslash$ $B$ or in $\bigcup_{j \geq n} T_{j} \backslash B$ and whose endpoints are in $T_{n} \backslash B$. The latter kind has its endpoints in the same component of $T_{n}$, by Lemma 13 . We join these endpoints by paths in $T_{n}^{\prime} \backslash F$. This leaves us with the problem of contracting finitely many loops that lie either in $\bigcup_{j \leq n} T_{j}^{\prime} \backslash F$ or in $\widetilde{K} \backslash H(E)$ with homotopies that miss $A$. Since the first kind can be homotoped into $s H(D) \subseteq \widetilde{K} \backslash H(E)$ with a homotopy that misses $A$, we are actually left with only loops of the second kind.

So, we now assume that $\gamma$ is a loop in $\widetilde{K} \backslash H(E)$ to be contracted missing $A$. We do this as before. Since $\widetilde{K}$ is simply connected, we can contract $\gamma$ to a point in $\widetilde{K}$. Let $\eta$ be the image of that contraction. Since $H$ is infinite, there is an $h \in H$ with $h(\eta) \cap A=\emptyset$. By choice of $E$ we can homotope $\gamma$ to $h \circ \gamma$ by a homotopy missing $H(A)$, where it contracts missing $A$.

6. Proof of Corollary A. Since we may assume by Theorem 2 that $N$ is at least 2-dimensional, we see that $\widetilde{N}$ is one-ended. Then $H=\pi_{1}(N)$ 
is one-ended and infinite. Finally, both $\widetilde{M} / H$ and $N$ are $K(H, 1)$ 's and are thus homotopy equivalent. Then $H_{n}\left(\widetilde{M} / H ; \mathbb{Z}_{2}\right)=H_{n}\left(N ; \mathbb{Z}_{2}\right)=0$. Therefore $\widetilde{M} / H$ is not compact. If we denote $\pi_{1}(M)$ by $G$, this implies that $G / H$, whose cardinality equals the number of sheets of the covering $\widetilde{M} / H \rightarrow M$, is infinite. Now apply Theorem 1 to complete the proof.

7. Proof of Theorem 3. Let $H$ be a subgroup of a group $G$. Suppose $L$ is a finite simplicial complex with regular covering $\widehat{L} \rightarrow L$ whose automorphism group is isomorphic to $G$. Denote the quotient $\widehat{L} / H$ by $\bar{L}$. We say that the pair $(G, H)$ is two-ended if for every compact set $A \subseteq \bar{L}$ there is a compact set $B \subseteq \bar{L}$ with $A \subseteq B$ such that $\bar{L} \backslash B$ has two components both of which are unbounded. It can be shown that this notion is independent of the choice of $\widehat{L} \rightarrow L$. (See [13] and [14] for a more general discussion of ends of pairs of groups.)

Proof of Theorem 3. Let $\widetilde{K}, \bar{K}, K, p, q$ be as in Section 5. Let $A \subseteq \widetilde{K}$ be a compact set. We will find a compact set $B \subseteq \widetilde{K}$ such that $A \subseteq B$ and $\widetilde{K} \backslash B$ is simply connected in $\widetilde{K} \backslash A$. Since $(G, H)$ is two-ended, we may choose a finite subcomplex $C_{1} \subseteq \widetilde{K}$ such that $A \subseteq C_{1}$ and $\bar{K} \backslash q\left(C_{1}\right)$ has two components both of which are unbounded. We also arrange for the inclusion induced map $\pi_{1}\left(q\left(C_{1}\right)\right) \rightarrow \pi_{1}(\bar{K})$ to be surjective and for $H\left(C_{1}\right)$ to be path connected. (Note that $\pi_{1}(\bar{K}) \simeq H$ is finitely generated.) Use Lemmas 4 and 5 to choose a finite subcomplex $C_{2} \subseteq \widetilde{K}$ such that $C_{1} \subseteq C_{2}$, $H\left(C_{1}\right)$ is one-ended in $H\left(C_{2}\right)$, and $H\left(C_{1}\right)$ is simply connected in $H\left(C_{2}\right)$. Again, we may assume that $\bar{K} \backslash q\left(C_{2}\right)$ has two components both of which are unbounded. Since the infinite group $\mathbb{N}_{G}(H) / H \simeq \operatorname{Aut}(\bar{K} \rightarrow K)$ acts properly discontinuously on $\bar{K}$ and $(g H)(q(T))=q(g(T))$ for all $g \in \mathbb{N}_{G}(H)$ and $T \subseteq \widetilde{K}$, there are elements $g_{1}, \ldots, g_{5} \in \mathbb{N}_{G}(H)$ such that the collection $\left\{q\left(g_{i}\left(C_{2}\right)\right) \mid i=1, \ldots, 5\right\}$ is pairwise disjoint and such that $q\left(g_{i}\left(C_{2}\right)\right)$ lies in the bounded component of $\bar{K} \backslash\left(q\left(g_{i-1}\left(C_{2}\right)\right) \cup q\left(g_{i+1}\left(C_{2}\right)\right)\right)$ for $i=2,3,4$. We take $g_{3}=1$.

Let $D$ be a finite subcomplex of $\widetilde{K}$ such that $q(D)$ equals the complement of the two unbounded components of $\bar{K} \backslash\left(q\left(g_{1}\left(C_{2}\right)\right) \cup q\left(g_{5}\left(C_{2}\right)\right)\right)$. By Lemma 8, there is a compact set $C_{3} \subseteq \widetilde{K}$ with $A \subseteq C_{3}$ and such that loops in $q^{-1}(q(D)) \backslash C_{3}=H(D) \backslash C_{3}$ can be homotoped into $q^{-1}\left(q\left(g_{2}\left(C_{1}\right)\right)\right)=$ $g_{2} H\left(C_{1}\right)$ missing $A$. Finally, we choose a compact set $B \subseteq \widetilde{K}$ with $C_{3} \subseteq B$ such that $g_{1} H\left(C_{1}\right) \backslash B$ and $g_{5} H\left(C_{1}\right) \backslash B$ are path connected in $g_{1} H\left(C_{2}\right) \backslash C_{3}$ and $g_{5} H\left(C_{2}\right) \backslash C_{3}$, respectively.

Now, let $\gamma$ be a loop in $\widetilde{K} \backslash B$. We may assume that $\gamma$ is an edge path in the 1 -skeleton of $\widetilde{K}$. If $\gamma$ lies outside of $H(D)$, we first contract it in $\widetilde{K}$. Since $g_{i} H\left(C_{1}\right)$ is simply connected in $g_{i} H\left(C_{2}\right)$, we can cut off this singular 
disk at $g_{2} H\left(C_{1}\right) \cup g_{4} H\left(C_{1}\right)$ and cap it off at $g_{2} H\left(C_{2}\right) \cup g_{4} H\left(C_{2}\right)$. Hence, in this case, $\gamma$ contracts missing $A$.

If $\gamma$ has subpaths which lie outside of $H(D)$ and whose endpoints are in $g_{1} H\left(C_{1}\right)$ or $g_{5} H\left(C_{1}\right)$, we connect the endpoints of each such subpath by a path in $g_{1} H\left(C_{2}\right) \backslash C_{3}$ or $g_{5} H\left(C_{2}\right) \backslash C_{3}$, respectively. Since we can deal with these newly formed loops as in the previous case, we may now assume that $\gamma$ lies entirely in $H(D) \backslash C_{3}$.

If $\gamma$ lies in $H(D) \backslash C_{3}$, we can homotope it into $g_{2} H\left(C_{1}\right)$ missing $A$, where it contracts within $g_{2} H\left(C_{2}\right)$, still missing $A$.

8. Davis's examples. In this section we will analyze the examples of Davis mentioned in the introduction, and discover that $\pi_{1}(N)$ equals its normalizer in $\pi_{1}(M)$ in these examples.

A Coxeter system $\Gamma=\langle V| v^{2}=1,(u v)^{m(u, v)}=1$ for all $\left.u, v \in V\right\rangle$ (a group defined in terms of finitely many generators and specific relations) is called right-angled if $m(u, v) \in\{\infty, 2\}$ for all $u \neq v$. Its nerve is defined to be the abstract simplicial complex $N(\Gamma, V)$ consisting of all non-empty subsets of $V$ which generate a finite subgroup of $\Gamma$, where incidence is by inclusion.

Let $P$ be the first barycentric subdivision of a non-simply connected PL-homology $(n-1)$-sphere. (Such examples exist in all dimensions 3 and higher.) Then there is exactly one right-angled Coxeter system $(\Gamma, V)$ whose nerve $N(\Gamma, V)$ is isomorphic to $P$, namely, the Coxeter group $\Gamma$ which is generated by the vertex set $V$ of $P$ and whose only relations are of the form $(u v)^{2}=1$ whenever $\{u, v\} \in P$ (cf. [4]). Let $C$ be the unique compact contractible $n$-manifold with boundary $N(\Gamma, V)$; it will serve as a basic chamber. Denote the dual cell of a vertex $v$ in $N(\Gamma, V)$ by $C_{v}$ (i.e. $C_{v}$ is the star of $v$ in a further barycentric subdivision of $N(\Gamma, V)$.) Put $\mathcal{M}(\Gamma)=\Gamma \times C / \sim$ where $(g, x) \sim(h, y) \Leftrightarrow x=y$ and $g^{-1} h \in\left\langle v \mid x \in C_{v}\right\rangle$. Then $\Gamma$ acts properly discontinuously and cocompactly on $\mathcal{M}(\Gamma)$ by left multiplication on the first coordinate.

In [3], Davis shows that $\mathcal{M}(\Gamma)$ is an open contractible manifold which is not homeomorphic to Euclidean space. It is well known that the commutator subgroup $[\Gamma, \Gamma]$ of $\Gamma$ is torsion free and of finite index in $\Gamma$. (To see this, consider the canonical epimorphism $\phi: \Gamma \rightarrow \mathbb{Z}_{2}^{|V|}$. Clearly, $[\Gamma, \Gamma] \subseteq \operatorname{ker} \phi$. Conversely, if $g \in \operatorname{ker} \phi$, then $g$ is the product of generators each appearing an even number of times. Hence, $g[\Gamma, \Gamma]=[\Gamma, \Gamma]$ in the abelian group $\Gamma /[\Gamma, \Gamma]$ so that $g \in[\Gamma, \Gamma]$. The index is consequently given by $[\Gamma:[\Gamma, \Gamma]]=[\Gamma$ : $\operatorname{ker} \phi]=\left|\mathbb{Z}_{2}^{|V|}\right|=2^{|V|}$. The fact that $[\Gamma, \Gamma]$ is torsion free can be proved using the right-angled reduction scheme below, as is done for example in Lemma 1.5 of [5].) Therefore $[\Gamma, \Gamma]$ acts fixed-point free and cocompactly on $\mathcal{M}(\Gamma)$. The quotient $\mathcal{M}(\Gamma) /[\Gamma, \Gamma]$ is our manifold $M$. 
Fix an element $v \in V$. Then $v$ acts as a reflection on $\mathcal{M}(\Gamma)$ through the fixed-point set $\operatorname{Fix}(v)=\{p \in \mathcal{M}(\Gamma) \mid v(p)=p\} . \operatorname{Put} \widetilde{V}=V \cap \operatorname{lk}(v, N(\Gamma, V))$ and let $(\widetilde{\Gamma}, \widetilde{V})$ be the induced right-angled Coxeter system. Then the nerve of $(\widetilde{\Gamma}, \widetilde{V})$ is the PL-sphere $\operatorname{lk}(v, N(\Gamma, V))$ and $\operatorname{Fix}(v)=\left\{g C_{v} \mid g \in \widetilde{\Gamma}\right\}$. In fact, we can identify $\operatorname{Fix}(v)$ with $\mathcal{M}(\widetilde{\Gamma})$, where $C_{v}$ takes on the role of the basic chamber. (See, for example, [6].) Now, $C_{v}$ is a ball, so that $\operatorname{Fix}(v)=\mathcal{M}(\widetilde{\Gamma})$ is homeomorphic to $(n-1)$-dimensional Euclidean space. Since the commutator subgroups, being the kernels of the respective abelianization homomorphisms, satisfy $[\widetilde{\Gamma}, \widetilde{\Gamma}]=[\Gamma, \Gamma] \cap \widetilde{\Gamma}$, the covering map $p: \mathcal{M}(\Gamma) \rightarrow$ $\mathcal{M}(\Gamma) /[\Gamma, \Gamma]=M$ restricts to $\left.p\right|_{\operatorname{Fix}(v)}: \operatorname{Fix}(v) \rightarrow \operatorname{Fix}(v) /[\widetilde{\Gamma}, \widetilde{\Gamma}]$. The quotient $\mathcal{M}(\widetilde{\Gamma}) /[\widetilde{\Gamma}, \widetilde{\Gamma}]$ is our manifold $N$. Clearly $\pi_{1}(N)=[\widetilde{\Gamma}, \widetilde{\Gamma}] \leq[\Gamma, \Gamma]=$ $\pi_{1}(M)$. We will now verify that in these examples $\mathbb{N}_{[\Gamma, \Gamma]}([\widetilde{\Gamma}, \widetilde{\Gamma}])=[\widetilde{\Gamma}, \widetilde{\Gamma}]$.

Recall that Coxeter groups have a very simple solution to the word problem [18]: a word (finite sequence of generators) is reduced (minimal in length) if and only if it cannot be shortened by a combination of the following two operations: (i) the obvious cancellation of a subword of the form $u$, and (ii) replacement of a subword of the form uwuwuw... (of length $m$ ) by wuwuwu... (of length $m$ ), where $m$ is the order of the element $u w$ in the group. This becomes especially easy to check in a right-angled Coxeter group. Specifically, if an element $g$ of a right-angled Coxeter group is expressed as a product of generators, say $g=u_{1} \ldots u_{q}$, then it can be brought into reduced form by repeated application of the following operation: deletion of some $u_{i}=u_{j}(1 \leq i<j \leq q)$ which commute with all of $u_{i+1}, \ldots, u_{j-1}$ in between, so that $g=u_{1} u_{2} \ldots \widehat{u}_{i} \ldots \widehat{u}_{j} \ldots u_{q}$, where the hat denotes omission.

Let $g \in[\Gamma, \Gamma]$ with $g[\widetilde{\Gamma}, \widetilde{\Gamma}] g^{-1}=[\widetilde{\Gamma}, \widetilde{\Gamma}]$. Express $g=u_{1} \ldots u_{q}$ reduced with all $u_{i} \in V$. We will show that $u_{i} \in \widetilde{V}$ for all $i$, so that $g \in[\widetilde{\Gamma}, \widetilde{\Gamma}]$. Choose the maximal index $i_{0}$ with $u_{i_{0}} \notin \widetilde{V}$ (if there is such an index). Since $[\widetilde{\Gamma}, \widetilde{\Gamma}]$ is a normal subgroup of $\widetilde{\Gamma}$, we have $u_{1} u_{2} \ldots u_{i_{0}}[\widetilde{\Gamma}, \widetilde{\Gamma}] u_{i_{0}} \ldots u_{2} u_{1}=[\widetilde{\Gamma}, \widetilde{\Gamma}]$. We now show that $u_{i_{0}} x=x u_{i_{0}}$ for all $x \in \widetilde{V}$. Let $x \in \widetilde{V}$. Choose $y \in \widetilde{V}$ with $x y x y \neq 1$. (The easiest way of seeing that such a $y$ always exists is to take $v$ to be a barycenter of a top-dimensional simplex in the original triangulation of the homology sphere.) Then

$$
u_{1} \ldots u_{i_{0}} x y x y u_{i_{0}} \ldots u_{1} s_{1} \ldots s_{p}=1
$$

for some $s_{i} \in \tilde{V}$, where we may assume $s_{1} \ldots s_{p}$ to be reduced. Applying the above right-angled reduction scheme to this equation, we conclude that $u_{i_{0}} x=x u_{i_{0}}$, because otherwise only five types of deletions would be possible:

(i) $u_{1} \ldots u_{i_{0}} x y x \widehat{y} u_{i_{0}} \ldots \widehat{u}_{j} \ldots u_{1} s_{1} \ldots s_{p}$ with $i_{0}>j \geq 1$;

(ii) $u_{1} \ldots u_{i_{0}} x y x \widehat{y} u_{i_{0}} \ldots u_{1} s_{1} \ldots \widehat{s}_{j} \ldots s_{p}$ with $1 \leq j \leq p$; 
(iii) $u_{1} \ldots \widehat{u}_{i} \ldots u_{i_{0}} x y x(y) u_{i_{0}} \ldots \widehat{u}_{j} \ldots u_{1} s_{1} \ldots s_{p}$ with $1 \leq i<i_{0}, i_{0}>j \geq 1$;

(iv) $u_{1} \ldots \widehat{u}_{i} \ldots u_{i_{0}} x y x(y) u_{i_{0}} \ldots u_{1} s_{1} \ldots \widehat{s}_{j} \ldots s_{p}$ with $1 \leq i<i_{0}, 1 \leq j \leq p$;

(v) $u_{1} \ldots u_{i_{0}} x y x(y) u_{i_{0}} \ldots \widehat{u}_{i} \ldots u_{1} s_{1} \ldots \widehat{s}_{j} \ldots s_{p}$ with $i_{0}>i \geq 1,1 \leq j \leq p$.

(The parentheses around $y$ in (iii) $-(\mathrm{v})$ denote the possibility of $y$ no longer occurring in this position, due to a previous deletion of type (i) or (ii).) However, no finite combination of these five reductions would ever cancel the word. (Notice that repeated application of any of these five types of deletions across the individually reduced expressions $u_{1} \ldots u_{i_{0}}, x y x y, u_{i_{0}} \ldots u_{1}$, and $s_{1} \ldots s_{p}$ will leave each expression individually reduced, because any reduction of the thus shortened expression could have been carried out in the original expression, where all relevant, but now missing, generators would have commuted with the would-be deletion pair.)

Since $u_{i_{0}} x=x u_{i_{0}}$ for all $x \in \tilde{V}$, we must have $u_{i_{0}}=v$, because $v$ is the only vertex of $N(\Gamma, V)$ which is joined to all vertices of its link. Inductively, we conclude that all $u_{i} \in\{v\} \cup \widetilde{V}$. Since the word $u_{1} \ldots u_{q}$ is reduced and contains every generator an even number of times, we have in fact $u_{i} \in \widetilde{V}$ for all $i$.

\section{References}

[1] M. Brown, The monotone union of open $n$-cells is an open $n$-cell, Proc. Amer. Math. Soc. 12 (1961), 812-814.

[2] A. Casson and D. Jungries, Convergence groups and Seifert fibered 3-manifolds, Invent. Math. 118 (1994), 441-456.

[3] M. W. Davis, Groups generated by reflections and aspherical manifolds not covered by Euclidean space, Ann. of Math. (2) 117 (1983), 293-325.

[4] -, Nonpositive curvature and reflection groups, in: Handbook of Geometric Topology, R. J. Daverman and R. B. Sher (eds.), North-Holland, 2002, 373-422.

[5] H. Fischer, Visual boundaries of right-angled Coxeter groups and reflection manifolds, Ph.D. thesis, Univ. of Wisconsin, Milwaukee, 1998.

[6] —, Boundaries of right-angled Coxeter groups with manifold nerves, Topology 42 (2003), 423-446.

[7] M. H. Freedman, The topology of four-dimensional manifolds, J. Differential Geom. 17 (1982), 357-453.

[8] D. Gabai, Convergence groups are Fuchsian groups, Ann. of Math. (2) 136 (1992), $477-510$.

[9] J. Hass, H. Rubinstein, and P. Scott, Compactifying coverings of closed 3-manifolds, J. Differential Geom. 30 (1989), 817-832.

[10] C. H. Houghton, Cohomology and the behaviour at infinity of finitely presented groups, J. London Math. Soc. (2) 15 (1977), 465-471.

[11] B. Jackson, End invariants of group extensions, Topology 21 (1982), 71-81.

[12] R. Lee and F. Raymond, Manifolds covered by Euclidean space, ibid. 14 (1975), $49-57$.

[13] G. P. Scott, Ends of pairs of groups, J. Pure Appl. Algebra 11 (1977), 179-198. 
[14] G. P. Scott and C. T. C. Wall, Topological methods in group theory, in: Homological Group Theory, C. T. C. Wall (ed.), London Math. Soc. Lecture Note Ser. 36, Cambridge Univ. Press, 1979, 137-203.

[15] L. C. Siebenmann, On detecting Euclidean space homotopically among topological manifolds, Invent. Math. 6 (1968), 245-261.

[16] J. Stallings, The piecewise-linear structure of Euclidean space, Proc. Cambridge Philos. Soc. 58 (1962), 481-488.

[17] G. A. Swarup, On the ends of pairs of groups, J. Pure Appl. Algebra 87 (1993), 93-96.

[18] J. Tits, Le problème des mots dans les groupes de Coxeter, in: Symposia Mathematica (INDAM, Rome, 1967/68), vol. 1, Academic Press, 1969, 175-185.

[19] C. T. C. Wall, Open 3-manifolds which are 1-connected at infinity, Quart. J. Math. Oxford Ser. (2) 16 (1965), 263-268.

[20] D. G. Wright, Contractible open manifolds which are not covering spaces, Topology 31 (1992), 281-291.

Department of Mathematical Sciences

Ball State University

Muncie, IN 47306, U.S.A.

E-mail: fischer@math.bsu.edu
Department of Mathematics Brigham Young University Provo, UT 84602, U.S.A. E-mail: wright@math.byu.edu

Received 25 February 2003;

in revised form 15 September 2003 